\title{
Preparation and Properties of Silicon- and Titanium-Containing Hybrid Nanocomposite Films Based on Ethyl Cellulose ${ }^{1}$
}

\author{
A. I. Suvorova ${ }^{a, *}$, A. R. Sharafeeva ${ }^{a}$, A. L. Suvorov ${ }^{b}$, I. S. Tyukova ${ }^{a}$, and B. I. Lirova ${ }^{a}$ \\ ${ }^{a}$ Ural Federal University, pr. Lenina 53, Yekaterinburg, 620000 Russia \\ ${ }^{b}$ Institute of Organic Synthesis, Ural Branch, Russian Academy of Sciences, \\ Akademicheskaya/S. Kovalevskoi ul. 20/22, Yekaterinburg, 620041 Russia \\ *e-mail: Anna.Suvorova@usu.ru \\ Received June 18, 2012; \\ Revised Manuscript Received February 6, 2013
}

\begin{abstract}
Nanocomposite hybrid films containing silicon and titanium compounds in the polymer matrix are prepared through the sol-gel method via the hydrolytic polycondensation of Si and Ti alkoxides (tetraethoxysilane and titanium tetrabutoxide) in the THF solution of a hydrophobic polymer, ethyl cellulose. Their structure and properties are studied with the use of a complex of physicochemical methods. During the hydrolysis of tetraethoxysilane and the subsequent polycondensation of the reaction products, silicon atoms are incorporated into the polymer and form $-\mathrm{O}-\mathrm{Si}-\mathrm{O}-$ bonds involving hydroxyl groups of ethyl cellulose. In the sol-gel method, titanium alkoxide yields nanosized particles of titanium dioxide that play the role of fillers in the polymer matrix. Titanium-containing films show solubility in THF and, after prolonged contact with the solvent, precipitate titanium dioxide from the solution. Hybrid films containing silicon are insoluble owing to the formation of a chemical network between polymer molecules and $\mathrm{Si}-\mathrm{OH}$ groups of the products of hydrolysis of silicon alkoxide, as confirmed by the IR data. It is shown that the amounts and types of alkoxides and the diameters of the structures formed in the polymer matrix via the sol-gel procedure affect the hydrophilicity levels of ethyl cellulose hybrid films and their abilities to swell in water and aqueous solutions of organic dyes (brilliant blue and methylene blue). Ethyl cellulose hybrid films are hydrophilic, and they facilitate the removal of dye molecules from aqueous solutions. The best properties are featured by the films containing nanosized particles of titanium dioxide in the polymer matrix.
\end{abstract}

DOI: $10.1134 / \mathrm{S} 0965545 \mathrm{X} 13070043$

One of the directions of the research activity of Anna Aleksandrovna Tager, who laid the basis for the development of polymer physical chemistry in the Urals, was the study of the properties and structures of multicomponent polymer systems containing dispersed fillers [1, 2]. These studies were of prime importance for practice. Recent decades have seen the emergence of new more energy efficient methods that allow the in situ incorporation of nanosized modifiers into polymers to improve the properties of the polymer system without the facilitation of phase separation during polymer exploitation. One of these methods is the sol-gel procedure, which allows the hydrolytic polycondensation of various alkoxides or halide derivatives of $\mathrm{Si}, \mathrm{Ti}, \mathrm{Zr}$, and other elements under mild conditions $\left(T<100^{\circ} \mathrm{C}\right)$ in polymer solutions. As a result, it is possible to prepare film or fiber organic-inorganic hybrid polymer materials that contain inorganic components in the form of nano- or microsized structural modifiers bound to the polymer matrix via intermolecular forces of various kinds: in some cases, via cova-

\footnotetext{
${ }^{1}$ This work was supported by the Russian Foundation of Basic Research, project no. 08-03-00552.
}

lent bonds [3, 4]. These nanocomposite materials are of interest for the creation of membranes, sorbents, and catalysts operating in gas, aqueous, and organicliquid media. The materials under consideration show promise for solving problems of environmental protection from various molecular, ionic, and fine pollutants [5-11]. Membranes based on cellulose diacetate are well known and are manufactured and employed in various countries [12]. However, practically no data are available on the use of cellulose ether-that is, ethyl cellulose (EC), which is a good film-forming polymer-for the preparation of membrane films suitable for the removal of diverse organic compounds (solvents, dyes, etc.) from aqueous media. Widening the scope of polymers for the design of materials useful for purification of colored aqueous outflows of textile, printing, paper, and other industries using dyes is still an urgent problem [13]. As was shown in [14, 15], Sicontaining organic-inorganic hybrid films show activity in the separation of water and organic solvents $[14,15]$. The use of hybrid polymer films with nanosized inorganic structural modifiers for the removal of organic compounds, including dyes, from aqueous solutions is of interest not only from the ecological 
Table 1. Characteristics of the used dyes

\begin{tabular}{l|l|l|l|}
\hline & $\mathrm{M}$ & $\lambda_{\max }, \mathrm{nm}$ & $\varepsilon, \mathrm{L} /(\mathrm{mol} \mathrm{cm}$ ) (in water) \\
\hline Brilliant blue & & \\
\hline
\end{tabular}

point of view but also owing to the need to recover these expensive compounds for the production cycle [13].

The goal of this study is to obtain hybrid organicinorganic films of EC (a hydrophobic polymer) containing compounds of not only silicon but also titanium as polymer modifiers via the sol-gel method. It was necessary to study the effect of the compositions of hybrid EC films that include compounds of Si or Ti on their structures; surface behaviors; hydrophilicity levels; and abilities to swell in aqueous media, including aqueous solutions colored with organic dyes. In addition, it was necessary to evaluate whether hybrid organic-inorganic films can be used for the removal of organic dyes from aqueous solutions.

\section{OBJECTS AND ANALYTICAL METHODS}

The research objects were nanocomposite organic-inorganic films prepared through the sol-gel method via the hydrolytic polycondensation of alkoxides in a 5\% solution of EC (brand K, type 100, a degree of substitution of 2.4 , and $M=1 \times 10^{5}$ ) in THF. The silicon and titanium alkoxides tetraethoxysilane (TEOS) $\mathrm{Si}\left(\mathrm{OC}_{2} \mathrm{H}_{5}\right)_{4}$ and titanium tetrabutoxide (TTB) $\mathrm{Ti}\left(\mathrm{OC}_{4} \mathrm{H}_{9}\right)_{4}$ were distilled before use. Colored aqueous media were modeled with the use of solutions of commercial dyes: Brilliant Blue R-250 (Serva) and Russian-produced methylene blue [16]. These dyes exhibit intense absorption (each with a maximum at a wavelength of $\lambda_{\max }$ ) and high molar extinction coeffi- cients $\varepsilon$ in the visible spectral region. The characteristics of the dyes are summarized in Table 1. The solutions of the dyes were prepared in deionized water purified on a standard membrane apparatus.

Organic-inorganic EC films were prepared via the sol-gel method at $50^{\circ} \mathrm{C}$ according to the procedure described in [14]. The ratio between alkoxide and the EC chain unit was varied from $1: 1$ to $4: 1(\mathrm{~mol} / \mathrm{mol})$ because, at larger amounts of alkoxides, brittle films were obtained. During the synthesis, hydrochloric acid $(0.15$ moles per mole of alkoxide [14, 17]) was used as a catalyst. The hydrolyzing agent was deionized water, which was added to the THF solution of EC and alkoxide in an amount of 6 moles per mole of TEOS or 3 moles per mole of TTB because, in an acidic medium, the excess of water may facilitate rapid hydrolysis of TTB to form titanium dioxide [18]. The synthesis was accompanied by an increase in the viscosity of the reaction mixture and formation of a thixotropic gel that decomposed under intense stirring. After 4.5-h synthesis, films were cast from the thick transparent reaction mass on a fluoroplastic substrate. The films were dried first for 3 days in a thermostat at $25^{\circ} \mathrm{C}$ and then for $48 \mathrm{~h}$ at $40-50^{\circ} \mathrm{C}$ in a vacuum oven until constant masses were attained. Drying conditions provided for the completion of the hydrolytic polycondensation of alkoxides. The thicknesses of the films were $100 \pm 5 \mu \mathrm{m}$. The compositions of the films were investigated via elemental analysis for $\mathrm{C}$ and $\mathrm{H}$ and $\mathrm{Si}$ and $\mathrm{Ti}$. (The latter analysis was performed via the weight method from the data on the combustion of 
Table 2. Amounts of silicon and titanium in the films

\begin{tabular}{l|c|c|c}
\hline $\begin{array}{c}\text { Initial film } \\
\text { components }\end{array}$ & $\begin{array}{c}\text { Alkoxide }: \mathrm{EC} \\
\text { unit, mol/mol }\end{array}$ & $\begin{array}{c}\text { Content of } \mathrm{SiO}_{2} \\
\text { or } \mathrm{TiO}_{2}, \%\end{array}$ & $\begin{array}{c}\text { Content of } \\
\mathrm{Si} \text { or Ti, \% }\end{array}$ \\
\hline TEOS-EC & $1: 1$ & 22.99 & 10.73 \\
& $2: 1$ & 35.58 & 16.72 \\
& $3: 1$ & 42.70 & 20.07 \\
TTB-EC & $1: 1$ & 44.52 & 20.78 \\
& $2: 1$ & 30.16 & 18.10 \\
& $3: 1$ & 31.95 & 19.17 \\
& $4: 1$ & 35.97 & 21.38 \\
& & 37.85 & 22.69 \\
\hline
\end{tabular}

films in a flow of oxygen at $800^{\circ} \mathrm{C}$.) The real contents of Si and Ti in the hybrid films are listed in Table 2.

The surface reliefs of the films were studied on an NTegra-Thermo (NT-MDT) scanning atomic-force microscope via the tapping method. An NSG-11 probe with a force constant of $2.5-10 \mathrm{~N} \mathrm{~m}^{-1}$ was used. The surface scan area was 2.5 to $5.0 \mu \mathrm{m}^{2}$; 3D-surface images of the films (topologies) were obtained. The mean-square diameters of structures formed on the surfaces were estimated from the topology data; the results of calculations were presented as film-surface histograms of the frequencies of detection of structures on the surfaces versus the diameters (in $\mathrm{nm}$ ) of these structures.

The interaction of EC with the products of hydrolysis and the subsequent polycondensation of alkoxides in the solution of EC was studied via FTIR spectroscopy. The spectra of the hybrid films were measured in the reflection mode in the range $500-4000 \mathrm{~cm}^{-1}$ on a Nicolet 6700 spectrometer. Both films dried after the synthesis and films swollen to equilibrium in aqueous solutions of dyes and dried again were examined.

The behaviors of hybrid films in colored aqueous solutions were studied on the basis of electronicabsorption-spectra measurements of changes that occurred in the concentrations of aqueous solutions of dyes as a result of film swelling in them. The spectra of solutions in the visible region (400-800 $\mathrm{nm}$ ) were recorded on a Helios- $\alpha$ spectrophotometer (Thermo Spectronic). Measurements were performed in temperature-controlled quartz cuvettes at $25^{\circ} \mathrm{C}$; deionized water was used as a reference liquid. The initial concentrations of the dye solutions in film-swelling experiments were $5 \times 10^{-5} \mathrm{~mol} / \mathrm{L}$ because previous studies with brilliant blue and methylene blue showed that the linear dependence of the optical density of a colored solution on concentration is fulfilled in the concentration range $(2-6) \times 10^{-5} \mathrm{~mol} / \mathrm{L}$ [19]. This finding made it possible to conduct a highly precise $( \pm 0.10-0.15 \%)$ estimation of the changes in the compositions of aqueous solutions of dyes once swelling of the films in them is completed.
The swelling of hybrid films in the aqueous solutions of dyes was investigated via the gravimetric method; changes in their masses were estimated (with a precision of $\pm 0.0002 \mathrm{~g}$ ) once the equilibrium degrees of swelling in the aqueous colored media were achieved. The surface areas of the films and the volumes of liquid over them in these experiments were the same for all films. The equilibrium degrees of swelling, $\alpha_{\text {eq }}$, of the films in colored aqueous solutions were determined gravimetrically from the kinetic curves of swelling. The degrees of swelling, $\alpha$, were calculated through the relationship $\alpha=\left(m-m_{0}\right) / m_{0}$, where $m_{0}$ and $m$ are the masses of the dry film and the film swollen for a certain time. The properties of hybrid films containing $\mathrm{Si}$ or Ti were compared on the basis of the values of $\alpha_{\mathrm{eq}}$. The masses of the dyes absorbed by hybrid films were measured for the samples dried after swelling to constant masses in air and then after heating in vacuum under the above-mentioned conditions providing for the removal of trace water from the swollen films.

The hydrophilicity levels of the films were determined from the parameters characterizing wetting of their surfaces with deionized water. The contact angles for water droplets at the film-surface/air boundary was calculated from the geometrical dimensions of the base and the height of 5-7 droplets that were measured with the use of a cathetometer (with a precision of up to $\pm 0.0005 \mathrm{~m}$ ) [20]. The error in contact-angle $\theta$ measurements did not exceed $\pm 0.2^{\circ}$. The cosine of the contact angle is the measure of wetting. From the value of $\cos \theta$ and the surface tension of water, $\sigma_{\mathrm{w}}$, at $25^{\circ} \mathrm{C}$, work of adhesion $W_{\mathrm{a}}$ of water to a film was calculated via the following relationship [20]:

$$
W_{\mathrm{a}}=\sigma_{\mathrm{w}}(1+\cos \theta) \text {. }
$$

The values of $W_{\mathrm{a}}$, like $\cos \theta$, characterize the hydrophilicity levels of the films.

\section{RESULTS AND DISCUSSION}

\section{Characteristics of the Surfaces of Hybrid Films}

Films of EC and all hybrid films were X-ray amorphous. Hybrid films containing silicon were transparent and sufficiently flexible, while Ti-containing films were slightly opalescent and more rigid than siliconcontaining films obtained at the same reagent ratios.

Figure 1 shows the surface topologies (3D images) for one of the compositions of EC hybrid films containing silicon (Fig. 1a) and one of the compositions of EC hybrid films containing titanium (Fig. 1b). Similar data were obtained for films of other compositions. Histograms of the structure size distribution on the surfaces of the same films (Figs. 1c, 1d) indicate that the diameters of structures, $d$, were higher in the case of hybrid films containing titanium. Thus, in the studied sample (Figs. 1a, 1c) containing Si, structures with diameters of up to $100 \mathrm{~nm}$ prevail, while the film con- 
(a)

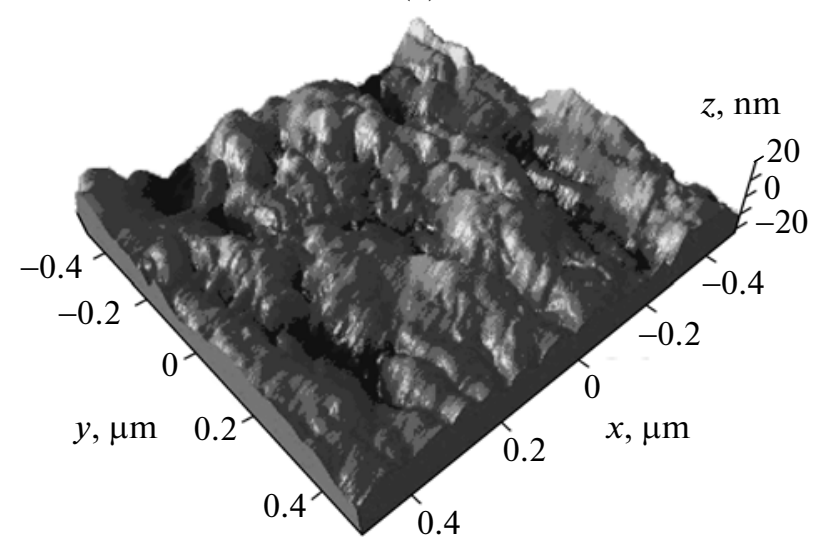

(b)

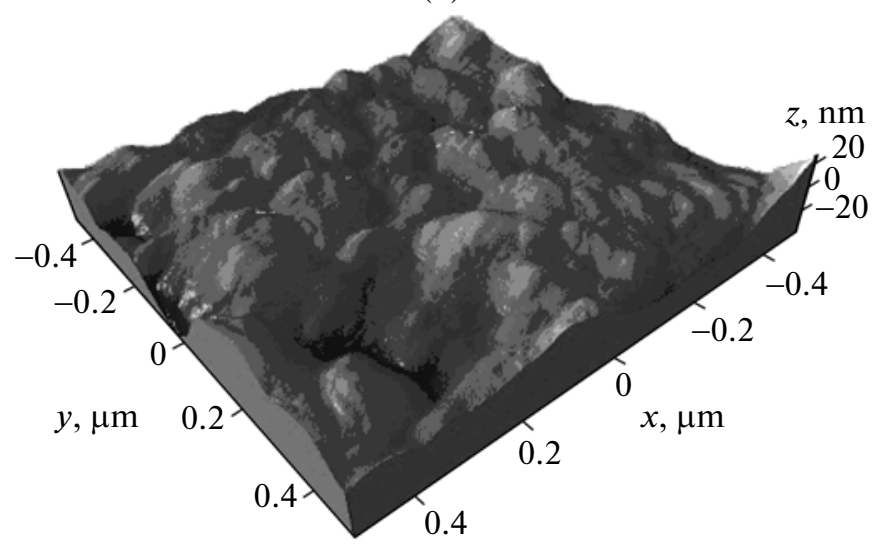

Fraction of structures

(c)

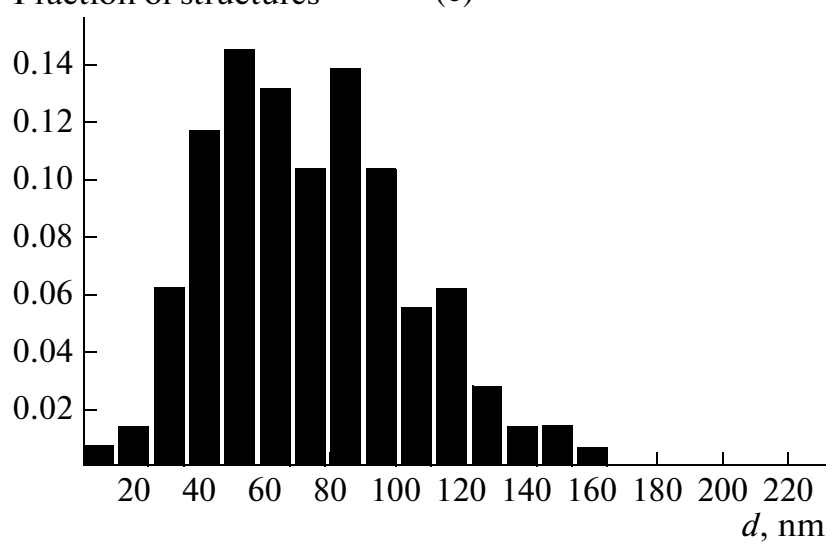

Fraction of structures

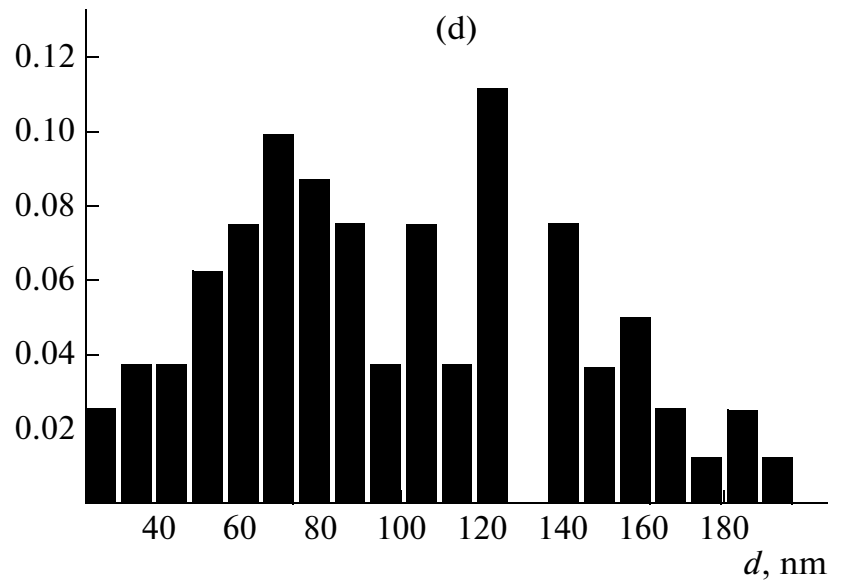

Fig. 1. (a, b) Surface topologies (3D image) and (c, d) dependences of the fractions of nanostructures of the film surfaces vs. their diameters $d$ for EC films containing $22 \%$ (a, c) Si and (b, d) Ti.

taining Ti contains a sufficient amount of structures with diameters of up to $180 \mathrm{~nm}$ (Figs. 1b, 1d). Similar data were obtained for EC hybrid films of other compositions: All films containing titanium had larger structures on the surfaces. As is known [20], the roughness of a surface influences the adhesion and sorption properties of a material.

\section{Interaction of Components Forming Hybrid Films}

To gain insight into the differences in the surface properties of EC-based hybrid films containing inorganic structural elements in the polymer matrix, the IR spectra of EC, alkoxides, and hybrid films were measured. The spectrum of EC (Fig. 2, curve 1) shows an intense band at $1050 \mathrm{~cm}^{-1}$, which corresponds to the stretching vibrations of $\mathrm{C}-\mathrm{O}-\mathrm{C}$ and $\mathrm{C}-\mathrm{OH}$ groups. There was a medium-intensity band at $3476 \mathrm{~cm}^{-1}$, which corresponds to vibrations of groups involved in the formation of intermolecular hydrogen bonds in the polymer. There were well-defined bands in the range $2870-2913 \mathrm{~cm}^{-1}$, typical for $\mathrm{C}-\mathrm{H}$ bonds in compounds carrying alkoxide radicals $\mathrm{C}_{2} \mathrm{H}_{5} \mathrm{O}$ [21], which are contained in units of EC chains. On passage to the EC hybrid films containing $\mathrm{Si}$, the spectrum demonstrates a gain in intensity of the low-frequency shoulder at the most intense band (at $1050 \mathrm{~cm}^{-1}$ ) and its shift to $\sim 950 \mathrm{~cm}^{-1}$. In addition, there is a slight broadening of the absorption band at $1050 \mathrm{~cm}^{-1}$, a phenomenon that may result from superposition of absorption bands due to $\mathrm{C}-\mathrm{O}-\mathrm{H}$ groups of the polymer and absorption bands due to stretching vibrations of $\mathrm{Si}-\mathrm{O}-\mathrm{C}$ and $\mathrm{Si}-\mathrm{O}-\mathrm{H}$ groups and symmetric stretching vibrations of disiloxane groups $\mathrm{Si}-\mathrm{O}-\mathrm{Si}$, which are formed during condensation of the products of hydrolysis of TEOS [17, 18]. In the high-frequency region $\left(3400-3500 \mathrm{~cm}^{-1}\right.$ ) for EC-Si films, there is a considerable widening of the band due to $-\mathrm{OH}$ groups of the polymer linked via intermolecular hydrogen bonds [21] and an increase in its intensity with an increase in the content of silicon in the EC film. All these facts provide evidence for the formation of strong H-bonds between unsubstituted hydroxyl groups of glucoside rings of EC and the products of 


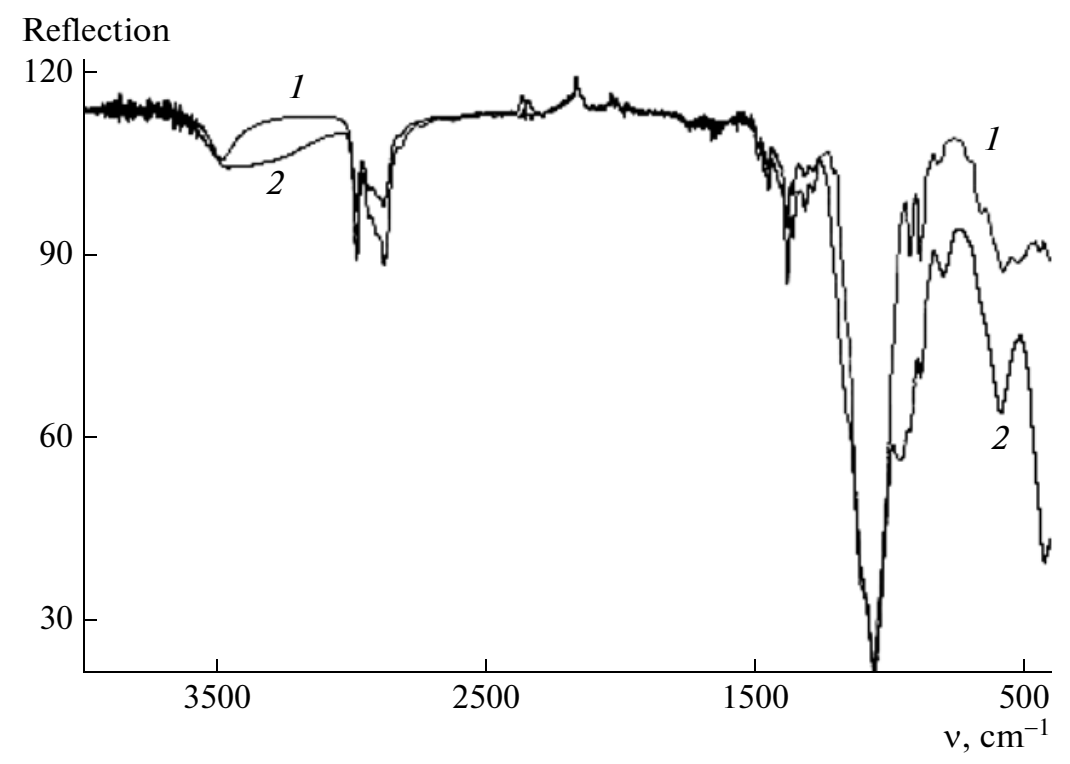

Fig. 2. IR spectra of (1) the EC film and (2) a hybrid film of EC-Si obtained at TEOS : EC unit = $4: 1(\mathrm{~mol} / \mathrm{mol})$.

hydrolysis of TEOS. It is not improbable that covalent bonds $-\mathrm{O}-\mathrm{Si}-\mathrm{O}-$ are formed between unsubstituted $-\mathrm{OH}$ groups of polymer chains and silanol $-\mathrm{Si}-\mathrm{OH}$ groups as a result of hydrolysis during the sol-gel synthesis. Unfortunately, the above-mentioned superposition of the bands due to the stretching vibrations of $\mathrm{C}-\mathrm{OH}$ groups of the polymer and silanol groups of the products of alkoxide hydrolysis cannot provide an unambiguous confirmation based only the IR spectra that the covalent organic-inorganic network of bonds is formed in EC films, as described by the scheme developed for similar reactions involving alkoxides in the sol-gel synthesis [4]. The presence of these bonds may be corroborated by the absence of solubility of $\mathrm{EC}-\mathrm{Si}$ hybrid films in THF, which is one of the best solvents for EC [22].

Compared to the change in the spectrum of the individual polymer EC, changes in the spectra of EC-Ti hybrid films are very slight (Fig. 3) and cover only the region corresponding to vibrations of $-\mathrm{OH}$ groups involved in the formation of intermolecular hydrogen bonds. At the same time, the IR spectrum of the dry residue isolated from the suspension formed after the dissolution of EC-Ti hybrid films in THF exhibits bands in the range $420-920 \mathrm{~cm}^{-1}$, which are attributed to titanium dioxide; these bands correspond to $\mathrm{O}-\mathrm{Ti}-\mathrm{O}$ bonds typical for the crystalline modification of titanium dioxide and oligomeric compounds of orthotitanates [23-25]. This result may lend support to the predominant hydrolysis of TTB to form the oxide during acidic catalysis under the conditions of the sol-gel synthesis. It is evident that the full hydrolysis of TTB via $\mathrm{Ti}-\mathrm{OC}_{4} \mathrm{H}_{9}$ bonds, accompanied by the formation of dioxide, is a thermodynamically more favorable reaction, while the interaction of titanoxane
$\mathrm{Ti}-\mathrm{OH}$ groups arising from hydrolysis with free $-\mathrm{OH}$ groups of ethyl cellulose does not occur. As a result, as in [15], titanium dioxide formed through the sol-gel process occurs in the EC polymer matrix in the form of nano- or microsized $\mathrm{TiO}_{2}$ particles that form intermolecular hydrogen bonds with the polymer. As follows from the comparison of absorption bands in the high-frequency region of the spectra (Figs. 2, 3; curves 2), their intensities in the EC-Ti films were much lower than those in the EC films containing Si. The spectral data make it possible to state that the products of hydrolysis and condensation of TEOS are incorporated into the polymer matrix owing to strong intermolecular and partially covalent bonds and that, in films prepared via hydrolysis of TTB in the solution of $\mathrm{EC}, \mathrm{TiO}_{2}$ particles that play the role of nanosized fillers in EC films are formed.

\section{Effects of Modifiers on the Hydrophilicity Levels of EC Hybrid Films}

The film of EC-cellulose ether-is poorly wet with water because, owing to the replacement of hydrogen atoms in - $\mathrm{OH}$ groups of the polymer with radical $-\mathrm{C}_{2} \mathrm{H}_{5}$, the polymer acquires hydrophobic properties. As a result, EC becomes insoluble in water [22]. The data on contact angles measured at the film/water/air interfacial boundary (Table 3 ) showed that, in the case of the EC film, contact angle $\theta$ for water droplets is above $90^{\circ}$; that is, the film of the individual polymer is hydrophobic. The incorporation of silicon and titanium compounds in the hybrid films causes decreases in the contact angles and increases in $\cos \theta$ and the energies of interaction of the films with water, $W_{\mathrm{a}}$, as is typical for improvement of the hydro- 


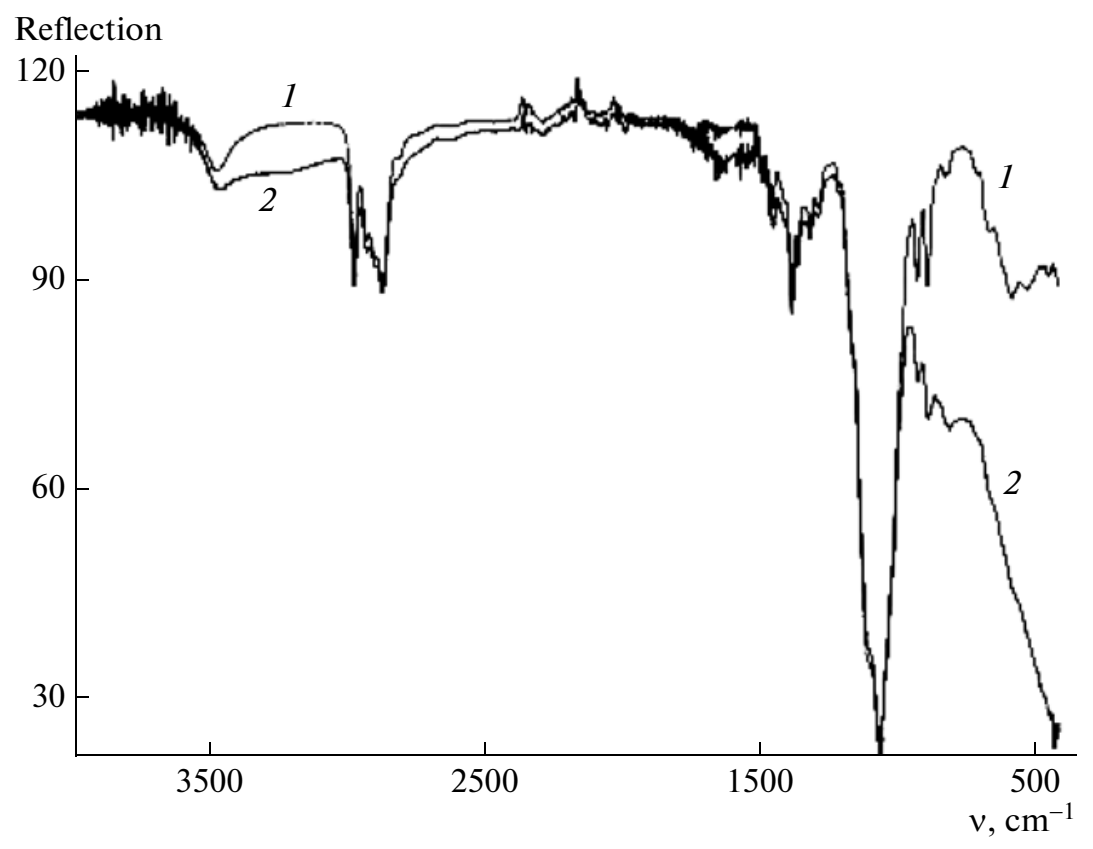

Fig. 3. IR spectra of (1) EC film and (2) a hybrid film of EC-Si obtained at TTB : EC unit = $4: 1(\mathrm{~mol} / \mathrm{mol})$.

philic behavior of a material [20]. As is seen from Table 3, for the $\mathrm{TiO}_{2}$-containing films of comparable compositions, the numerical values of $\cos \theta$ and $W_{\mathrm{a}}$ are somewhat higher than those for EC-Si films. This tendency may be explained not only by the difference in the nature of the used modifier but also by the diameters of structures formed the surfaces of films. As can be seen from the AFM data (Figs. 1c, 1d), the diameters of nanostructures on the surfaces of EC-Ti films are higher than those on the surfaces of EC-Si films. It is well known [20] that the properties of a solid surface, specifically its roughness, affect its wetting. The common result of incorporation of silicon or titanium compounds in the films of the hydrophobic polymer EC is that the hydrophilicity levels of the surfaces are improved with increases in the amounts of $\mathrm{Si}$ and $\mathrm{Ti}$ modifiers in the films; therefore, these films are applicable for operation in aqueous media.

\section{Removal of Dyes from Aqueous Solutions by Hybrid Films of EC}

The use of hybrid organic-inorganic films of EC to remove organic dyes from aqueous solutions is of interest for practice. Therefore, the swelling of hybrid films in aqueous solutions of dyes was investigated not only through a kinetic study of swelling to determine the equilibrium degree of swelling, $\alpha_{\text {eq }}$, but also with the use of spectrophotometry via measurements of changes in the concentrations of dyes in colored solutions due to attainment of the equilibrium state by the films. As was noted in [13], the latter phenomenon is important for estimation of the secondary use of waste dye solutions.

Figure 4 plots the dependence of $\alpha_{\mathrm{eq}}$ on the amounts of modifiers in the hybrid films of EC swollen in dissolved dyes. The hydrophobic film of EC free of inorganic modifiers slightly swells in water and dye solutions. The presence of Si and Ti structural modifiers in the films facilitated gains in hydrophilicity and, accordingly, in the abilities of films to swell in aqueous media: The higher the content of the inorganic modifier in a film, the more pronounced the increase in $\alpha_{\mathrm{eq}}$. In this case, the incorporation of $\mathrm{TiO}_{2}$ into films immediately causes sharp increases in the abilities of

Table 3. Characteristics of wetting of EC hybrid films with water

\begin{tabular}{l|c|r|r|c}
\hline Film & $\begin{array}{c}\text { Alkoxide-to-EC- } \\
\text { unit molar ratio }\end{array}$ & $\cos \theta$ & $\theta, \mathrm{deg}$ & $\begin{array}{c}W_{\mathrm{a}}, \\
\mathrm{mJ} / \mathrm{m}^{2 *}\end{array}$ \\
\hline $\mathrm{EC}$ & 0 & -0.04 & 104.0 & 69.48 \\
$\mathrm{EC}-\mathrm{Si}$ & $1: 1$ & 0.13 & 82.5 & 81.79 \\
$\mathrm{EC}-\mathrm{Si}$ & $2: 1$ & 0.25 & 75.5 & 90.48 \\
$\mathrm{EC}-\mathrm{Si}$ & $3: 1$ & 0.35 & 69.5 & 97.71 \\
$\mathrm{EC}-\mathrm{Si}$ & $4: 1$ & 0.40 & 66.5 & 101.33 \\
$\mathrm{EC}-\mathrm{Ti}$ & $1: 1$ & 0.17 & 80.0 & 83.96 \\
$\mathrm{EC}-\mathrm{Ti}$ & $2: 1$ & 0.30 & 72.5 & 94.09 \\
$\mathrm{EC}-\mathrm{Ti}$ & $3: 1$ & 0.38 & 67.0 & 99.88 \\
$\mathrm{EC}-\mathrm{Ti}$ & $4: 1$ & 0.44 & 63.0 & 104.2 \\
\hline
\end{tabular}

Note: $*$ Calculated at a surface tension of water of $\sigma=72.38 \mathrm{~mJ} / \mathrm{m}^{2}$. 


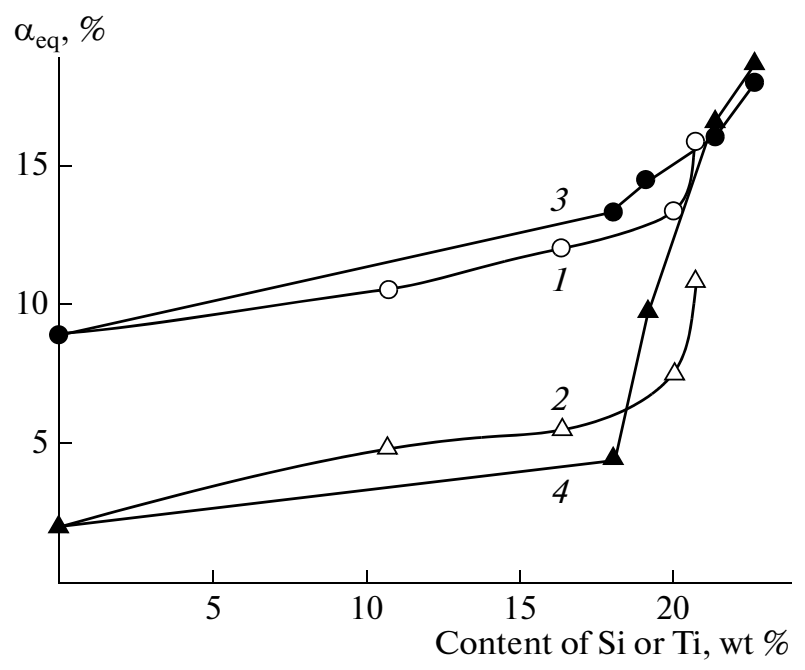

Fig. 4. Effects of the quantitative compositions of $(1,2)$ $\mathrm{EC}-\mathrm{Si}$ and $(3,4) \mathrm{EC}-\mathrm{Ti}$ films on their equilibrium degrees of swelling in aqueous solutions of $(1,3)$ brilliant blue and $(2,4)$ methylene blue.

the films to swell in solutions of both dyes (Fig. 4, curves 3,4). For films containing Si atoms chemically incorporated into the structure of EC, gradual increases in the swelling abilities of the films are observed. A more intense swelling of the films in solutions of butyl alcohol (at a content of $\mathrm{Si}$ or $\mathrm{Ti}<20 \mathrm{wt} \%$ ) levels off at higher amounts of the modifiers. This finding is apparently associated with attainment of the optimally necessary loosening of the polymer structure, which occurs faster if nanosized particles of titanium dioxide $\mathrm{TiO}_{2}$ are incorporated into the structure of EC during the sol-gel synthesis. In the same composition range, as is seen from Table 3, the values of parameters characterizing the hydrophilicity levels of the films come close. Changes occurred in the types of surfaces and in the inner structures of the films at modifier contents of $\sim 20 \%$ (these compositions are obtained during synthesis at alkoxide-to-polymer-unit molar ratios of 0.75 and 1.0). As a consequence, the set of properties of the films changed significantly.

Spectrophotometric measurements of the concentrations of dye solutions before the onset of swelling of the films in them and after completion of this process revealed reductions in the intensities of coloration of solutions. Large molecules of organic dyes (Table 1) do not penetrate inside the films; they can only adsorb on the surfaces of glassy hybrid films of EC from solution, as confirmed by the visual analysis of the cross sections of dried films, which were colored on the surfaces and were colorless in their inner parts. In contrast, small water molecules easily penetrate inside hybrid films with hydrophilic properties and cause their swelling in aqueous solutions; the concentrations of dye solutions remain unchanged in this case.

On the basis of spectrophotometric data on the concentrations of dye solutions measured before and

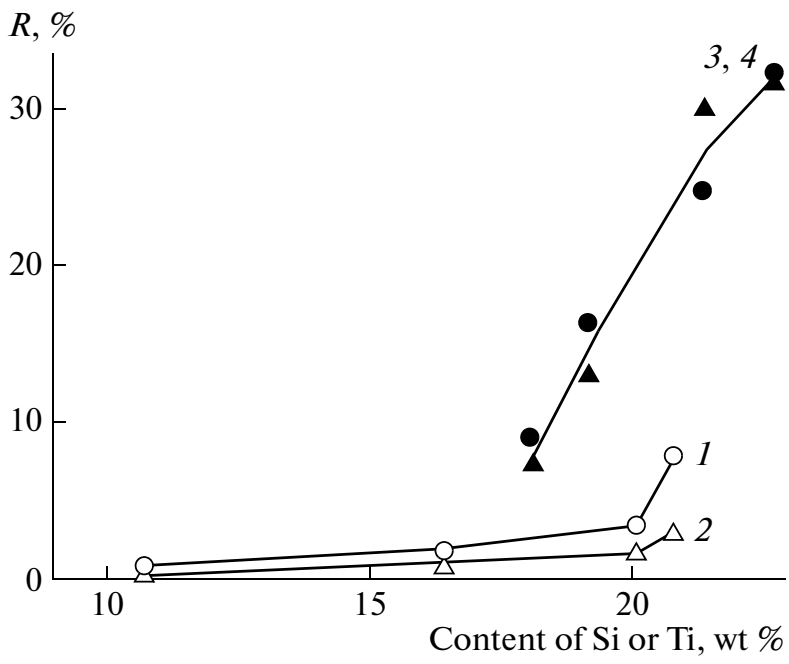

Fig. 5. Effects of the composite films of EC on changes in the concentrations of aqueous solutions of dyes during swelling of $(1,3)$ brilliant blue and $(2,4)$ methylene blue films. $(1,2) \mathrm{EC}-\mathrm{Si}$ and $(3,4) \mathrm{EC}-\mathrm{Ti}$ in solutions.

after equilibrium swelling in the given solutions of hybrid films of EC, selectivity $R$ of the effects of films on the compositions of solutions were evaluated. The values of $R$ were calculated via the following relationship [26]:

$$
R=\left(1-\frac{C_{\mathrm{eq}}}{C_{0}}\right) \times 100 \%,
$$

where $C_{0}$ is the initial concentration of a dye solution, which was the same in all experiments $\left(5 \times 10^{-5} \mathrm{~mol} / \mathrm{L}\right)$, and $C_{\mathrm{eq}}$ is the concentration of a dye in a solution after completion of film swelling. The latter value gradually decayed during swelling of a hybrid film with an intensity depending on the amount and type of the modifier incorporated into the film. Because $C_{\mathrm{eq}}<C_{0}$ and $C_{0}$ is a constant value in all experiments, a decrease in the concentration of a solution after film swelling in it leads to an increase in $R$, in accordance with relationship (2). Figure 5 illustrates how changes in the compositions of the films containing Si- or Ti-based modifiers influence the values of $R$, which characterize the efficiencies of changes in the concentrations of dye solutions as a result of swelling of hybrid films in them. As the amount of $\mathrm{Si}$ or $\mathrm{Ti}$ in a film increases, $R$ tends to increase; that is, the concentration of a solution changes more efficiently. Marked changes in $R$ were observed in the case of films containing a modifier in the form of nanosized particles of $\mathrm{TiO}_{2}$ (Fig. 5, curves 3,4$)$, which promote these changes to the highest extent. It appears that the films modified through interaction of the polymer with $\mathrm{Si}-\mathrm{OH}$ groups of the products of hydrolysis are denser; the presence of the chemical network hinders their swelling in water. Therefore, the dye concentration due to swelling of an EC-Si film in solution changes to a considerably 
weaker extent (Fig. 5, curves 1, 2). However, the pattern of the dependence of $R$ on film composition (Fig. 5) makes it possible to infer that the EC-Si films show a tendency toward an increase in efficiency at a higher content of silicon compounds in the polymer matrix.

\section{CONCLUSIONS}

The above evidence shows that EC-based hybrid films containing $\mathrm{Si}$ or Ti inorganic modifiers incorporated into the polymer matrix via the sol-gel method change their internal structures and surface behaviors under the action of the modifiers. As a result of modification, the films based on hydrophobic polymer EC acquire hydrophilicity not only in the case of incorporation of silicon atoms into the polymer via formation of $\mathrm{Si}-\mathrm{O}-\mathrm{C}$ chemical bonds between macromolecules and the products of hydrolysis of silicon alkoxide and but also in the presence of nanosized $\mathrm{TiO}_{2}$ particles dispersed in the polymer matrix that appear via the acidic hydrolysis of titanium alkoxide during the solgel synthesis. The resulting hydrophilic films containing compounds of Si and Ti swell in water, as opposed to the films based on individual hydrophobic polymer EC. The parameters determining the hydrophilicity levels of hybrid films increase with an increase in the weight ratio of either type of modifier introduced in $\mathrm{EC}$, but the effect is more intense if the polymer contains nanosized $\mathrm{TiO}_{2}$. It has been found that, owing to the acquisition of hydrophilic properties, organicinorganic hybrid films of EC that contain compounds of silicon or titanium may be employed in aqueousorganic solutions. As has been shown for solutions of the two organic dyes (brilliant blue and methylene blue, which exhibit high coloration intensities), during swelling in aqueous solutions of dyes, the tested films facilitate reductions in their concentrations. This phenomenon is of importance for use in practice.

The body of the above-described properties of hybrid films in the studied composition range suggests that the EC films containing titanium oxide in the form of nanosized particles dispersed in the matrix swell better and operate better in aqueous and aqueous-organic media. This circumstance may be used in the removal of various organic pollutants (dyes, solvents) from aqueous and aqueous-organic solutions [13, 27], a process that may be important for protecting the environment.

\section{ACKNOWLEDGMENTS}

We are grateful to A.V. Ivliev for his help in measuring and processing of AFM surface images of hybrid films.

\section{REFERENCES}

1. A. A. Tager, S. M. Yushkova, Yu. S. Bessonov, V. V. Guzeev, M. N. Rafikov, and V. S. Ezhev, Vysokomol. Soedin., Ser. A 21, 1051 (1979).
2. S. M. Yushkova, A. A. Tager, Yu. S. Bessonov, V. V. Guzeev, and V. S. Ezhev, Vysokomol. Soedin., Ser. A 24, 1373 (1982).

3. T. Uragami, K. Okazaki, H. Matsugi, and T. Miyata, Macromolecules 35, 9156 (2002).

4. T. Uragami, H. Matsugi, and T. Miyata, Macromolecules 38, 8440 (2005).

5. D. Tian, Ph. Dubois, and R. Jerome, J. Polym. Sci., Part A: Polym. Chem. 35, 2295 (1997).

6. M. Y. Kariduraganavar, S. S. Kulkarni, and A. A. Kitter, J. Membr. Sci. 246, 83 (2005).

7. R. Guo, Ch. Hu, F. Pau, H. Wu, and Z. Jiang, J. Membr. Sci., No. 3, 254 (2006).

8. S. Sh. Rashidova, D. Sh. Shakarova, O. N. Ruzimuradov, et al., J. Chromatogr. 800, 49 (2004).

9. D. Anjali Devi, B. Smitha, S. Sridhar, and T. M. Aminabhavi, J. Membr. Sci. 262, 91 (2005).

10. S. Sequeira, D. V. Evtuguin, Y. Portugal, and A. P. Esculcas, Mater. Sci. Eng. C 27, 172 (2007).

11. R. A. Zoppi and M. C. Goncalves, J. Appl. Polym. Sci. 84, 2196 (2004).

12. A. I. Schäfer, A. G. Fane, and T. D. Waite, Nanofiltration. Principles and Application (Elsevier, Amsterdam, 2005).

13. Yu. P. Osadchii and A. V. Nevskii, Ekol. Prom. Ross., No. 3, 46 (2009).

14. A. I. Suvorova, A. L. Suvorov, M. V. Ivanenko, and E. I. Shishkin, Ross. Nanotekhnol. 4, 154 (2009).

15. A. I. Suvorova, I. S. Tyukova, and A. L. Suvorov, Glass Phys. Chem. 37, 629 (2011).

16. F. I. Sheikhet, Materials Science of Chemicals, Dyes and Detergents (Legkaya Industriya, Moscow, 1969) [in Russian].

17. M. G. Voronkov, Siloxane Bond (Nauka, Novosibirsk, 1976) [in Russian].

18. Y. Yang and P. Wang, Polymer 47, 2683 (2006).

19. Fundamentals of Analytical Chemistry. Methods of Chemical Analysis. (A Manual for High School), Ed. by Yu. A. Zolotov (Vysshaya Shkola, Moscow, 2004) [in Russian].

20. B. D. Summ, Fundamentals of Colloid Chemistry (Akademiya, Moscow, 2006) [in Russian].

21. L. J. Bellamy, The Infra-Red Spectra of Complex Molecules (Methuen, London, 1954).

22. Polymers: A Property Database, Ed. by B. Ellis and R. Smith (CRC, Taylor and Francis Group, Boca Raton, 2009), p. 155.

23. G. A. Semerneva, A. L. Suvorov, and L. A. Samarina, Zh. Prikl. Spectrosk. 3, 555 (1965).

24. N. Agoudjil and T. Benkacem, Desalination 206, 531 (2007).

25. I. S. Ignatyev, M. Montejob, and J. J. L. Gonzalez, Vibr. Spectrosc. 59, 218 (2009).

26. A. A. Svittsov, An Introduction to Membrane Technology (De Li print, Moscow, 2007) [in Russian].

27. V. V. Parashchuk and A. V. Volkov, Ser. Krit. Tekhnol. Membr., No. 31, 25 (2006).

Translated by T. Soboleva 